\title{
Complementariedades disciplinares entre la Diplomática y la Documentación a partir del proceso de Análisis Documental: elementos para una reflexión
}

\author{
Discipline complementarities between Diplomatics and Documentation \\ starting from the process of Documental Analysis: elements for a reflection
}

Rodrigo RABello (1) y José Augusto Chaves Guimarães (2)

(1) Becario de la Fundação de Amparo a Pesquisa do Estado de São Paulo (FAPESP). Rua Tabajara, 71 Senador Salgado Filho, CEP: 17502-610 - Marília-SP, rdgrabello@yahoo.com.br. (2) Profesor Titular e Investigador del Conselho Nacional de Desenvolvimento Científico e Tecnológico (CNPq). Av. Hygino Muzzi Filho, 737 Caixa postal 420 - Marília-SP, 55 14-3402-1302. E-mail: guimajac@marilia.unesp. br.

\section{Resumen}

Se analizan las relaciones disciplinares entre Diplomática y Documentación a partir del proceso de análisis documental.

Palabras clave: Diplomática. Documentación. Análisis documental. Interdisciplinariedad.

\section{Introducción}

La Diplomática y la Documentación encuentran en el documento su principal preocupación investigativa. De esa forma, esas disciplinas son relevantes por permitir la reflexión acerca del fenómeno documental que, de manera general, consiste en el proceso de producción, procesamiento y apropiación del conocimiento registrado como apoyo para la construcción de un nuevo conocimiento.

Sin embargo, el mismo interés en la cuestión documental que las acerca también las separa caso consideremos las preocupaciones de orden teórico-metodológico que se constituyeron de manera singular en el desarrollo de ambas disciplinas.

Nuestro objetivo es realizar una revisión teórica acerca de las particularidades de esas disciplinas teniendo como eje una preocupación marcadamente interdisciplinar, en el sentido de localizar y señalar posibles intersecciones de diálogo en el ámbito conceptual y de los procesos documentales en cuestión.

Con esa finalidad, hemos recurrido a autores que investigaron los aspectos histórico-contextuales de ambas disciplinas, enfatizando sus enfoques teórico-prácticos originales y también su proceso de constitución académico-formal.

\begin{abstract}
The interdisciplinary relations between Diplomatics and Documentation are analyzed, with an special attention to the document analysis process.
\end{abstract}

Keywords: Diplomatics. Documentation. Document analysis. Interdisciplinarity.

La investigación ha mostrado el desarrollo de los distintos enfoques y de las metodologías metodológicas en el campo documental, principalmente en el universo de la organización y representación de la información y del conocimiento en que se inserta disciplinarmente el Análisis Documental. La comprensión de esos enfoques ha sido relevante para la proposición de un posible diálogo entre Diplomática y Documentación.

\section{Aspectos histórico-disciplinares de la Diplomática y de la Documentación}

Autores como Bauer (1957), Valente (1978), Duranti (1995), Rabikauskas (2000) y Rondinelli (2002) han demostrado las sutilezas del proceso de formalización académica de la crítica documental (siglo XVII) rumbo a la disciplinarización de la Diplomática (siglo XIX). Ese proceso se caracterizó por el desarrollo del método diplomático en su diversidad de aplicaciones en distintas disciplinas, tales como la Historia, el Derecho y la Archivística, entre otras.

La Diplomática se originó como disciplina de los estudios filológicos y teológicos desarrollados a partir del perfeccionamento de la "crítica documental", que durante el siglo XVII ya presentaba las primeras iniciativas en dirección a la "sistematización metódica" y a la búsqueda del "rigor 
científico" de la actividad de distinguir documentos falsos de los verdaderos (Valente, 1978).

Según explican Duranti (1995) y Rabikauskas (2000), la palabra "diplomática" deriva del voca-

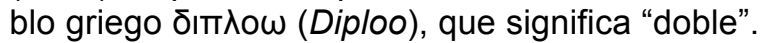
Para Duranti (1995), la Diplomática sería una adaptación del latín de la obra De re diplomatica libri VI, escrita por el monje francés Jean de Mabillon. Esa obra fue escrita, mediante un trabajo metódico, con el objetivo de aclarar las reglas fundamentales de la crítica textual $y$, particularmente, de responder a las críticas diplomáticas realizadas por el jesuita Daniel Van Papenbroek, quien cuestionó la autenticidad de los diplomas beneditinos que se encontraban en los archivos de la Orden de San Bento. Como afirma Duranti (1995, p. 23), la obra de Mabillon representa "[...] el primer escrito sobre ese tema refiriéndose al análisis crítico de las formas del diploma".

Tales estudios fundaron una fase marcada por la búsqueda de cientificidad para el proceso de crítica documental, teniendo por fin fundamentarla en un método "objetivo" para la realización de tests de autenticidad y de veracidad de los documentos.

La inserción de la Diplomática en la academia ocurrió en una fase posterior. Esta fase se inició con la búsqueda de la cientificidad cuando aquella disciplina alcanzó (juntamente con la Paleografía) la categoría de "ciencia auxiliar" de la Historia y del Derecho. La creación de la École des Chartes, en París, precisamente en el año 1821, se constituyó como referencia de esa misma fase. Tal institución fue importante para que se estableciera un eslabón entre la instrumentalidad de la Diplomática a la Historia, pues su método era aplicado a los documentos de archivo (documentos medievales; escritos y manuscritos, por lo tanto), que eran las fuentes documentales "por excelencia" del historiador.

Así pues, la École fue relevante para la aproximación entre Diplomática y Archivística, la cual ocurrió, en gran medida, mediante la instrumentalización del método diplomático, que posibilitó su empleo para la gestión de los documentos archivísticos en la segunda mitad del siglo XX (Duranti, 1995)

En ese proceso "evolutivo", podemos decir que mientras la Diplomática "clásica" históricamente se interesó por la práctica (y técnica) de identificar y describir los elementos formales (estructurales) de los documentos escritos con la finalidad de verificar la autenticidad y fidelidad documental; con el empleo del método diplomático en los archivos se añadió a la Diplomática una función instrumental distinta. A partir de esa relación interdisciplinar, se originó, en el siglo $X X$, la Diplomática Contemporánea —también conocida como Diplomática Archivística o Tipología Documental (Duranti, 1995; Bellotto, 2002)

Entonces, el interés de la Diplomática se vuelve hacia la "génesis documental", convergiendo y contextualizándose en las atribuciones, competencias, funciones y actividades de la entidad generadora o acumuladora. Su objeto de estudio es el "tipo documental" que trae consigo la "lógica orgánica de los conjuntos documentales"; al contrario de la Diplomática "clásica", que tiene como objeto la "especie documental", es decir, el documento en su ejemplar único (Bellotto, 2002, p. 19-20).

El documento diplomático, en ese contexto, comenzó a ser concebido como sinónimo de documento archivístico, en el que destacan los valores histórico y jurídico-administrativo (Heredia, 1988; Riesco Terrero, 2000; Bellotto, 2000). Ese documento se caracteriza, como lo describen Guimarães (1994) y Bellotto (2002), por dos elementos nucleares: los elementos (o caracteres) "externos" (también conocidos como extrínsecos, físicos, estructurales), relativos a la forma, a la apariencia del documento; y los elementos "internos" (también conocidos como intrínsecos, sustantivos o de sustancia), relativos al contenido del ( $y$ en el) documento.

Esa disciplina cuenta con el método diplomático para el estudio de tales elementos documentales. De modo genérico, el método diplomático se caracteriza como la estrategia de identificación de fórmulas documentales (en distintas especies y tipologías) que busca establecer relaciones particulares entre forma y contenido. Más claramente, podemos afirmar que el método diplomático presenta un camino para la identificación de aquello que los lingüistas han definido como macroestructura textual o macroproposición semántica.

Respecto a las cuestiones disciplinares de la Documentación, es interesante destacar que el término "documentación", a lo largo de la Historia, asumió diferentes connotaciones, tales como: a) medio de prueba histórica; b) profesión enseñada formalmente en escuelas técnicas, y que demanda un saber técnico-intelectual especializado; y c) disciplina académica dedicada a la creación de saberes que inspiran las prácticas documentales (Meyriat, 1993).

Reflexionando sobre el último aspecto, mencionado por Meyriat, la Documentación se originó a partir del movimiento bibliográfico del final del siglo XIX (Woledge, 1983; Blanquet, 1993; López Yepes, 1995; etc.); y se constituyó disciplinarmente en el inicio del siglo XX, marcada 
por la publicación del Traité de Documentation, escrito por el abogado belga Paul Otlet, en 1934.

Entre los siglos XVIII y XIX, la elaboración de las bibliografías especializadas (de carácter científico) acompañaba el amplio desarrollo de las ciencias, ganando mayores proporciones en un momento en que eran elaboradas con el patrocinio de asociaciones de investigadores. Ese fue el contexto en que no solamente fueron perfeccionadas las técnicas bibliográficas, sino también nació "[...] un campo disciplinar que, a lo largo de la Historia, recibe diversas denominaciones, de las cuales las más conocidas son Biblioteconomía, Documentación y Ciencia de la Información" (Santos, 2006, p. 13).

De acuerdo con Rayward (1994, p. 237), las bases del movimiento bibliográfico otletiano que originó disciplinarmente la Documentación fueron definidas en el ensayo intitulado Something about Bibliography, escrito por Paul Otlet en 1892. En ese texto, el autor argumentó que la función de la Bibliografía consistía en auxiliar en la identificación y organización del material bibliográfico mediante la sistematización del proceso de análisis temático y organización bibliográfica. Rayward (1994, p. 237) destaca aún que el movimiento bibliográfico otletiano fue el "primer paso" para la creación de un amplio sistema de procesamiento documental (documentary processing) que posteriormente sería la base para la creación de una red internacional de documentación.

Según exlica Blanquet (1993, p. 201), sumada a la importante actividad de análisis temático de los documentos dirigida a la organización bibliográfica, se inició con esos trabajos una ruptura en el campo de la información, pues como la función principal del movimiento bibliográfico consistía en "memorizar" el conocimiento registrado, tal iniciativa, aunque en forma despropositada, ofreció parámetros importantes (como la función de "difusión" y de "acceso" a la información) para la creación disciplinar de la Documentación.

Otro factor influyente para su constitución fueron las innumerables publicaciones, reuniones científicas, asociaciones y foros de debates académicos creados y articulados por Otlet, como fue el caso de la Oficina Internacional de Bibliografía (OIB), creada en 1892, y del Instituto Internacional de Bibliografía (IIB), creado en 1895, los cuales fueron relevantes para la creación del Repertorio Bibliográfico Universal (RBU), que sería un instrumento que permitiría el acceso del conocimiento registrado producido en ámbito internacional (Otlet, 1934, p. 381). La organiza- ción del conocimiento registrado sería posible mediante su clasificación previa por medio de la Clasificación Decimal Universal (CDU), creada a partir de la Clasificación Decimal del estadounidense Melvil Dewey (CDD), que actuaría como instrumento para formar la "enciclopedia del saber" (Ibidem, p. 381).

La Documentación se distinguió de la Bibliografía por una función primordial: la difusión de la información desde una concepción del documento que transcendió la noción de documento bibliográfico (Rayward, 1995). En otras palabras, para Otlet el documento no se limitaba al concepto "libro", es decir, a aquel objeto que resultó de un perfeccionamento técnico del códice medieval tras la creación de los tipos móviles gutemberguianos. Para Otlet, el documento era todo objeto producido (objetivado) por el hombre que cumplía una función informativa, incluyendo los contenidos documentales expresados en diferentes soportes y formatos (fotografías, mapas, etc.). Tal concepción innovadora fue el punto de partida para que la bibliotecaria francesa Suzanne Briet (1951) ampliara aún más ese concepto. A partir de su obra, podemos decir que el acto interpretativo del sujeto/sociedad (movido por una necesidad informativa) frente al objeto (natural o manufacturado/industrializado) sería la acción definidora de la "transformación" del objeto en documento (Rabello, 2006).

Como destacan López Yepes (1990, p. 40) y Rayward (1994, p. 237-238), el Traité fue la sistematización de los estudios de Otlet sobre las cuestiones relativas al libro y al documento, en particular, en lo que se refiere a la organización, recuperación y disponibilización de la información, es decir, a todos los elementos informacionales que componen el universo de la Documentación.

Disciplinarmente, según Otlet (1937), la Documentación "[...] acompaña el documento desde el instante en que éste surge de la pluma del autor hasta el momento en que impresiona el cerebro del lector", asumiendo pues un carácter pasivo, receptivo o dativo en diversos sectores de la sociedad. (1)

Por lo tanto, el desarrollo disciplinar de la Documentación fue, en gran medida, asociado al desarrollo de las actividades de colecta documental desde un enfoque metódico y técnico que buscó reunir, clasificar y organizar explorando las potencialidades informativas de los documentos (Briet, 1951), tratando de construir de ese modo un sistema técnico-social (Meyriat, 1981). 


\section{Complementariedades entre Diplomática y Documentación a partir del Análisis Documental}

Si pensamos la Documentación como la disciplina científica promotora de un sistema técnicosocial (Meyriat, 1981) —cuyas necesidades informacionales (individual/social) definen la configuración del sistema de gestión del conocimiento registrado- poderemos reflexionar, en ese momento, acerca del posible diálogo entre Diplomática y Documentación, tomando como referencial las actividades que se insertan en el universo teórico de la Organización y de la Representación de la Información y del Conocimiento, en particular, en las prácticas disciplinares del ámbito del denominado Tratamiento Temático de la Información (TTI) - (2) que marcaron un período de búsqueda del perfeccionamento técnico-documental.

El desarrollo del TTI ocurrió a partir de la necesidad pragmática del tratamiento documental, reflejando aquello que Smit (1986, p. 12) describió como reunir y organizar para encontrar la información documental. Según explica Barité (1998, p. 124), el TTI "[...] tiene como objeto los aspectos vinculados al análisis, a la descripción y a la representación del contenido de los documentos, así como sus inevitables interfaces con las teorías y los sistemas de almacenamiento y recuperación de la información". Dada su naturaleza mediadora entre la producción y el uso de la información, Guimarães (2006) observó que el TTI viene construyendo disciplinarmente, a lo largo de los tiempos, su referencial teórico-metodológico, especialmente a partir de tres corrientes: la catalogación de asunto (subject cataloguing), de influencia estadounidense; la indexación (indexing), de influencia inglesa; y el análisis documental (analyse documentaire), de influencia francesa.

En el ámbito de los estudios en Documentación, el Análisis Documental (AD) encontró su origen en Francia, a partir del final de la década de los sesenta, particularmente, con los trabajos de Jean-Claude Gardin y de Maurice Coyaud. Dichos estudios tenían la inherente preocupación de desarrollar referenciales teórico-metodológicos para el proceso de identificación del contenido documental. El tema impulsor de tal movimiento deriva del hecho de que, hasta entonces, esas operaciones eran orientadas por procedimientos la mayoría de las veces empíricos, con criterios diversificados y de naturaleza subjetiva. Por lo tanto, la proposición del AD marcó el momento en el que se buscaron parámetros que otorgasen alguna cientificidad, encontrando en la explicitación de los procedimientos o de los mecanismos documentales en cuestión el punto de apoyo de su misma concepción. Según destacan Gardin et al. (1981, p. 29), los procedimientos en $A D$ pasan, necesariamente, por contribuciones oriundas especialmente de la Lingüística, de la Lógica y de la Terminología.

Históricamente, el propio contexto de origen de la Documentación evidencia el motivo central de la posterior proposición del $A D$, principalmente si se considera que la producción documental impresa en el siglo XIX se expandió notablemente y fue en el ámbito de la especialización del conocimiento científico que el periódico se presentó como un importante instrumento social de divulgación científica y de ideas, debiendo igualmente ser urgentemente organizado y tratado en otro paradigma teórico y conceptual que transcendiera el de la Biblioteconomía "clásica". Fue con la Documentación que se comenzó a considerar la pluralidad de asuntos contenidos en los distintos artículos de los periódicos mediante el instrumental de la CDU. En esa perspectiva, Fayet-Scribe (2001, p. 14) mostró que con esa disciplina el libro dejó de ser concebido como uno y comenzó a ser desmembrado (de su conjunto) su contenido documental, interesando ya no el libro en sí mismo, sino los asuntos contenidos en él.

Partiendo de la conjetura de que la percepción otletiana de la multiplicidad temática contenida en el documento fuera el punto inicial para el desarrollo teórico-metodológico de la perspectiva francesa del AD; y considerando las posibles contribuciones normativas e instrumentales que el método diplomático puede propiciar al proceso de análisis documental del contenido de los documentos, buscaremos trazar posibles puntos de convergencia entre Documentación y Diplomática en el ámbito del TTI.

De modo particular, la actividad de $A D$ se inserta en los límites del trinomio que impregna el universo informacional —producción, organización y uso-, y por el propio orden y disposición de tal proceso, la actividad de organización actúa y se destaca como aquélla que ocupa una posición mediadora. En el contexto de la Documentación, la organización busca rescatar los elementos documentales (informaciones) que permitan su identificación y recuperación, pudiendo enfatizar los aspectos documentales extrínsecos, de naturaleza formal; o intrínsecos, relativos a aquello que se convencionó designar "contenido informacional". Destacamos la imprescindibilidad de tales aspectos para que la información (materializada en el documento) pueda ser recuperada y posteriormente utilizada, pudiendo propiciar la generación de nuevas informaciones. 
En el ámbito de la organización (o tratamiento documental), Kobashi (1994) destaca que tanto el soporte material como el contenido constituyen aspectos esenciales del documento, cada cual de ellos demandando respectivamente distintas formas de tratamiento. Específicamente en el horizonte del tratamiento documental del contenido reside el objeto del Análisis Documental (AD), el cual busca, por fin, "[...] propiciar la elaboración de representaciones condensadas del contenido informacional del documento a partir del contexto en que se inserta" (Guimarães y Rabello, 2007, p. 145). En ese campo de acción, dos productos se presentan: el primero, de naturaleza más analítica y textual, denominado de inventario y de resumen (en archivos y bibliotecas/centros de documentación), y el segundo, de naturaleza más sintética: el índice.

Los productos del $A D$ recién destacados son fruto de un "[...] conjunto de procedimientos efectuados con la finalidad de expresar el contenido de los documentos bajo formas destinadas a facilitar la recuperación de la información" (Smit, Guimarães, 2001); resultantes de una etapa analítica propiamente dicha, o sea, de un "análisis dentro del análisis" (Guimarães, 2003); y otra, ulterior, de síntesis, ya mediante una condensación (resúmenes), ya por medio de la representación en lenguajes documentales (encabezamientos de asunto, notaciones clasificatorias, descriptores de tesauros), con objeto de mejorar la precisión en el proceso de recuperación de la información.

De este modo, de esas dos etapas deriva el objetivo particular del AD, al considerarse, según argumenta Guimarães (2003), la existencia de un encadenamiento lógico de procedimientos de decomposición de un registro informacional en sus elementos constitutivos. Podemos decir que con las etapas analítica y sintética se busca el sentido informativo del documento, acompañado de una abreviada "reconstrucción" (representación) del documento en un momento en que se vuelven evidentes sus puntos de mayor expresividad temática. En rigor, esas actividades son impulsadas por el interés de hacer disponible el contenido informacional para que llegue efectivamente al usuario de forma ágil y adecuada a su necesidad.

El proceso de identificación y de selección del contenido documental (resultante de la etapa analítica) se vehicula a partir del procedimiento previo de "lectura documental" y, principalmente, de un procedimiento de identificación de conceptos (Guimarães, 2003). En esa etapa, el analista dispone de diversas estrategias metacognitivas de análisis, entre las cuales el méto- do diplomático desempeña un papel destacado, lo cual evidencia el efectivo carácter instrumental de la Diplomática para el AD.

Tal carácter instrumental puede ser atribuido al método diplomático, en particular, a partir de la estrategia de identificación de fórmulas mediante la partición diplomática que puede ser traducida, como señalan Tessier (1952), NuñezContreras (1981), Bellotto (1991) y Tamayo (1996), entre otros, como la identificación de protocolos (o áreas documentales de estructura fija y previsible) que reflejan las relaciones de articulación de contenidos inherentes al documento. La posibilidad de visualización de la distinción entre forma y contenido, derivados del proceso de identificación de fórmulas que constituyen las macroestructuras textuales, transforman el método diplomático en un importante instrumental para el proceso de AD. (Guimarães, Rabello, 2007, p. 148).

La experiencia de investigación de Guimarães (1998) sobre el tema produjo importantes resultados que atestiguan esa instrumentalizad, pues fue posible demostrar la aplicabilidad del método diplomático para el tratamiento del contenido de sentencias judiciales. Después de comparar las especificidades dictadas por las tipologías documentales, el referido autor propuso principios de análisis que posibilitaron el delineamento de tres premisas orientadoras del método: a) cada documento nace con una función principal; b) documentos que miran por las mismas funciones presentan una fórmula (estructura) igual y específica; y c) la misma estructura puede servir para otros usos documentales distintos de su función original. Por lo tanto, Guimarães (1998, p. 100) postuló que la instrumentalidad del método diplomático para el tratamiento del contenido documental auxilia en la identificación de una trilogía de elementos: "función", "estructura" y "uso" del documento, lo cual contribuye a establecer los elementos de contenido que pueden permitir rescatarlo y organizarlo.

En ese contexto de complementariedad entre Diplomática y Documentación, la definición de la institucionalización del documento dependerá de los intereses de los usuarios potenciales del sistema de información. O sea, su uso definirá la forma de tratamiento y "lugar/ambiente" del documento. Por ejemplo, si se hiciera una solicitación documental para fines administrativos o de prueba (jurídica o histórica), la función y la procedencia del documento serían priorizadas en un abordaje archivístico. Si el énfasis se pusiera en el contenido temático, el documentalista igualmente tendría el auxilio del método diplomático para evaluar cómo la articulación de los elementos documentales (contenido, función 
y estructura) puede servir para generar productos documentales apropiados y/o personalizados.

El cuestionamento de la función básica de la estructura y de los usos subsidiarios del documento puede llevar al escrutinio o a la sedimentación del documento en sus diferentes protocolos (descriptivos y temáticos), entre los cuales se constituyen el eje instrumental del método diplomático para la identificación y representación del contenido documental en contextos variados. (Guimarães, Rabello, 2007, p. 149).

El carácter instrumental del método diplomático respecto al $A D$ puede ser observado en los proyectos de invetigación realizados en diversos contextos archivísticos y bibliotecarios, en los últimos años, en UNESP - Marilia, sobre documentos como Isentencias judiciales (Guimarães, 1994), etiquetas de shampoos (Straioto, 1997), recetas culinarias (Bueno, 1998), actas de reuniones administrativas (Guimarães, 1999), etiquetas de vinos (Maia, 2002), manuales de instrucción de equipos agrícolas (Reis, 2003) y prospectos de medicamentos (Sarde, 2003), entre otros.

A partir de tales estudios diplomáticos, Guimarães y Rabello (2007, p. 150-151) nos llevan a pensar sobre la complementariedad disciplinar ocurrida en la intersección de dos momentos: (1) el método diplomático actúa en la detección, sistematización y registro de las diferentes especies documentales, pudiendo consolidar, como conseqüencia, las distintas fórmulas diplomáticas, al permitir una transparencia mayor de las diferentes acciones desarrolladas por los órganos generadores de la documentación; (2) sirve como soporte para el proceso de análisis documental de contenido, suministrando una estrategia metacognitiva de lectura y minimizando los efectos de la subjetividad (o de los distintos cuadros de referencia) del analista.

De este modo, en el proceso de $A D$, el método diplomático se configura como un relevante instrumento para la determinación del contenido informacional de los documentos, en particular, para la detección de lo que Begthol (1986) denomina "tematicidad" (aboutness), al aliarse con los recientes desarrollos teórico-prácticos en la esencia de la Documentación relacionados a la imperiosa necesidad de la normalización terminológica en el proceso de análisis y síntesis de la información presentes en los estudios en AD (Smit, Guimarães, 1998).

La relación de complementariedad entre Diplomática y Documentación es perceptible, todavía, a partir de la intersección de, por un lado, los valores documentales "históricos" (testimo- nio o prueba histórica) y "jurídico-administrativos" (eminentemente de prueba para el Derecho), los cuales interesan principalmente a la Diplomática y a la Archivística; y, por el otro, con los valores de "conocimiento" y de "información", que en gran medida interesan a la Biblioteconomía y a la Documentación (Rabello, Guimarães, 2006).

Tales valores se suman a la importante función de socialización del conocimiento registrado, en la cual el proceso de organización se presenta como nuclear (Barité, 2001; Hjørland, 2003; Rabello, Guimarães, 2006), y en él tiene sentido la aproximación interdisciplinar entre Diplomática y Documentación. En ese contexto, tanto en el enfoque de la Diplomática como en el de la Documentación, serían contemplados aspectos relativos a la recuperación de la memoria (colectiva e individual), que podría ser identificada, respectivamente, a partir de la función de prueba histórica del documento típicamente archivístico; y en la esencia de la función documental de adquisición de los conocimientos históricamente inscritos, cuando son investigados en esos términos en un centro de documentación.

\section{Consideraciones finales}

La aproximación entre Diplomática y Documentación puede configurarse en una provechosa relación de complementariedad, si consideramos la importancia instrumental del método diplomático para el proceso de AD. Como vimos, uno de los principales objetivos de la Documentación es el de explorar las potencialidades informativas del documento (o sea, su contenido temático, mediante el $\mathrm{AD}$ ) con la finalidad de organizar los documentos en un sistema de información. De este modo, la Diplomática Contemporánea puede contribuir con la Documentación, si esta última disciplina no pasa por alto en sus procesos documentales el principio diplomático de que la función distintiva de un documento escrito se relaciona con su contenido temático, el cual, a su vez, se redacta bajo las reglas de una estructura documental específica (caracteres internos y externos). En un sentido inverso, el $A D$ puede contribuir a la Diplomática para la constitución de un proceso de orden lógico-lingüístico direccionado a la representación temática del contenido documental.

En ese sentido, observamos que la concepción diplomática de tipo documental trajo consigo una inclinación a la comparación de un documento con los demás del mismo tipo, caracterizándolos por su función, estructura y uso (Guimarães, 1998). A partir de esa comparación, la observación de la existencia o ausencia 
de elementos estructurales contribuiría a la comprensión de los aspectos de la macroestructura textual, proporcionando informaciones relevantes para su representación descriptiva. Sumándose a eso, la aplicación del método diplomático puede agregar un conjunto de recursos informacionales al proceso de tratamiento y representación temática propuesta por el AD.

Además de eso, la urgencia de un diálogo mayor entre Diplomática y Documentación podría ser observada, aún, en el contexto de la recuperación de la información en ambientes digitales, situación que vendría a sumarse y a contribuir con investigaciones que buscan en la diplomática soportes teórico-metodológicos para su uso en tales ambientes, como es el caso de la investigación de Duranti (1995), desdoblada en investigaciones como las de Rondinelli (2002), Nascimento (2002) y Furlaneto Neto (2003), entre otras.

Por fin, el presente texto ha buscado contribuir, en alguna medida, para que la proximidad procesual entre Diplomática y Documentación fuese evidenciada en un sentido contrario a la lógica de la especialización académica, que algunas veces dificulta ese posible y fructífero diálogo interdisciplinar. Como afirma Riesco Terrero (2000, p. 92), "[...] la Documentación y la Diplomática, en modo alguno pueden ignorarse, máxime, si se tiene en cuenta que los principios básicos de esta última estaban ya desarrollados mucho antes de nacer el documentalismo".

\section{Notas}

(1) El concepto disciplinar de Documentación de Otlet (1934) había sido retomado y, en cierta medida, profundizado por Briet (1951) y por teóricos como Meyriat (1993), Blanquet (1993), López Yepes (1995), Rayward (1995), Buckland (1997), entre otros.

(2) Según destacó Guimarães (2006), el TTI puede, históricamente, ser caracterizado en tres momentos: el arte -formas artesanales en el abordaje temático de la información, como por ejemplo los sobres de arcilla que describían el contenido de papiros y pergaminos en Mesopotamia, etc.-; la técnica -que, como hemos comentado, abarca los sistemas de clasificación-; y la búsqueda por metodologías - fase de la consolidación académica de la Biblioteconomía y, posteriormente, de la Documentación, que tiene como precursores a Kaiser (EUA, 1911), Ranganathan (India, 1933), Classification Research Group (Inglaterra, 1952), Derek Austin (Inglaterra, 1968) y al grupo de Bangalore (India, 1969).

\section{Referencias}

Barité, M. (1998). Referenciales teóricos vigentes en el área de tratamiento temático de la información y su expresión metodológica. Porto Alegre, ABEBD, 1998. // [Relatório técnico do II Encontro de Dirigentes dos cursos superiores de Biblioteconomia dos países do Mercosul, Buenos Aires, 27-29 nov. 1997].

Barité, M. (2001). Organización del Conocimiento: un nuevo marco teórico-conceptual en Bibliotecología y Documentación. // Carrara, Kester. Educação, universidade e pesquisa. Marília: Unesp, 2001. 35-50.

Bauer, W. (1957). Documentos. // Bauer, W. Introducción al estudio de la História. Trad. Luis G de Valdeavellano. 3.ed. Barcelona: Bosch, 1957.

Begthol, C. (1986). Bibliographic classification theory and text linguistics: aboutness analysis, intertextuality and the cognitive act of classifying documents. // Journal of Documentation. 42:2 (1986) 84-113.

Bellotto, H. L. (1991). Arquivos permanentes: tratamento documental. São Paulo: T. A. Queiroz, 1991

Bellotto, H. L. (2000). Diplomática. // Smit, J. W.; Lima, Y. D. Organização de arquivos: XIV Curso de especialização. São Paulo, Escola de Comunicação e Artes/USP, 2000. 50-68. (Gênese Documental, 2).

Bellotto, H. L. (2002). Como fazer análise diplomática e análise tipológica de documento de arquivo. São Paulo: Arquivo do Estado / Imprensa Oficial do Estado, 2002.

Blanquet, M.-F. (1993). La fonction documentaire: etude dans une perspective histórique. I/ DocumentalisteSciences de I'Information, 30:4-5 (1993) 199-204.

Briet, S. (1951). Qu'est-ce que la Documentation? Paris, EDIT, 1951.

Buckland, M. K. (1997). What is a document? // Journal of the American Society for Information Science. 48 (1997) 804-809.

Bueno, R. M. (1998). Receitas culinárias: uma aplicação do método diplomático. Monografia (Trabalho de Conclusão de Curso de Biblioteconomia). Marília: Universidade Estadual Paulista, 1998.

Duranti, L. (1995). Diplomática: usos nuevos para una antigua ciencia. Trad. Manuel Vászquéz. Carmona: S \& C Ediciones, 1995.

Fayet-Scribe, Sylvie (2001). Histoire de la documentation en France: culture, science et technologie de l'information: 1895-1937. Paris: CNRS, 2001

Furlaneto Neto, Mário. (2003). Pornografia infantil na Internet: elementos diplomáticos como subsídios à caracterização do delito. Dissertação (Mestrado em Ciência da Informação). Marília: Faculdade de Filosofia e Ciências, Universidade Estadual Paulista, 2003.

Gadin, J.-C. et al. (1981). La logique du plausible: essais d'épistemologie pratique. Paris, Ed. Maison des Sciences de l'Homme, 1981.

Guimarães, J. A. C. (1994). Análise documentária em jurisprudência: subsídios para uma metodologia de indexação de acórdãos trabalhistas brasileiros. Tese (Doutorado em Ciência da Comunicação). São Paulo: Escola de Comunicação e Artes, Universidade de São Paulo, 1994.

Guimarães, J. A. C. (1998). O caráter instrumental da Diplomática para tratamento temático de documentos na área jurídica. // Cadernos da F. F.C., Marília, 7:1-2 (1998) 97-106.

Guimarães, J. A. C. (1999). A Diplomática como perspectiva metodológica de tratamento documentário para o profissional da informação. // III Congreso de Archivologia del MERCOSUL, Montevidéu, 1999. (conferência). 
Guimarães, J. A. C. (2003). A dimensão teórica do Tratamento Temático da Informação (TTI) no universo científico da "International Society for Knowledge Organization" - ISKO. Marília, [s.n.]. (Projeto de Produtividade em Pesquisa (PQ) apresentado ao Conselho Nacional de Desenvolvimento Científico e Tecnológico - CNPq).

Guimarães, J. A. C.; Rabello, R. (2007). A contribuição metodológica da diplomática para a análise documental de conteúdo em arquivos e bibliotecas. // Richter, E. I. S.; Araujo, J. C. G. (dir.). Paleografia e Diplomática no curso de Arquivologia - UFSM. Santa Maria, FACOS UFSM, 2007. 137-157.

Heredia Herrera, A. (1988). Diplomática y Arquivística. // Arquivística General: teoria y practica. Sevilla: Diputación Provincial, 1988.

Hjørland, B. (2003). Fundaments of Knowledge Organization. // Know. Org. 30:2 (2003) 87-111.

Kobashi, N. Y. (1994). A Análise Documentária no âmbito da circulação de informação. // Kobashi, N. Y. A elaboração de informações documentárias: em busca de uma metodologia. Tese (Doutorado em Ciência da Comunicação). Escola de Comunicação e Artes, Universidade de São Paulo, São Paulo, 1994. 15-41.

Lopes, I. L. Organização e representação do conhecimento na perspectiva da Ciência da Informação. Brasília: Thesaurus, 2003. 100-117

López Yepes, J. (1995). La documentación como disciplina: teoria e história. 2. ed. PaMplona: EUNSA, 1995.

Maia, V. L. (2002). Elementos temáticos em rótulos de vinho: uma aplicação da abordagem facetada. Monografia (Trabalho de Conclusão de Curso em Biblioteconomia). Marília: Faculdade de Filosofia e Ciências, Universidade Estadual Paulista, 2002.

Meyriat, J. (1981). Document, documentation e documentalogie. // Revue de Bibliologie, Schema et Schematisation. 19 (1981) 51-63.

Meyriat, J. (1993). Un siècle de documentation: la chose et le mot. // Documentaliste-Sciences de I'Information. 30:4-5 (1993) 192-198.

Nascimento, L. M. B. do. (2002). A dimensão diplomática do documento jurídico digital. Dissertação (Mestrado em Ciência da Informação). Marília: Faculdade de Filosofia e Ciências, Universidade Estadual Paulista, 2002.

Núñez Contreras, L. (1981). Concepto de documento. // Archivistica: estudios básicos. Sevilla: Diputación Provincial, 1981. 25-44.

Otlet, P. (1937). Documentos e documentação: discurso pronunciado no Congresso de Documentação Universal, Paris, 1937. Rio de Janeiro, Imprensa Nacional (Separata). www.conexaorio.com/biti/otlet (2002-1-8).

Otlet, Paul (1934). Traité de documentation: le livre sur le livre: théorie et pratique. Bruxelles: Mundaneum, 1934.

Rabello, R. (2006). O documento na Ciência da Informação: em busca da construção de um objeto a partir das concepções da Documentação e da Diplomática. Marília, [s.n.], 2006. (Projeto de doutorado em Ciência da Informação - FFC-UNESP)

Rabello, R.; Guimarães, J. A. C. (2006). A relação conceitual entre conhecimento e documento no contexto da Organização do Conhecimento: elementos para uma reflexão. // VII Encontro Nacional de Pesquisa em Ciência da Informação (ENANCIB): a Dimensão Epistemológica da Ciência da Informação e suas Interfaces Técnicas, Políticas e Institucionais nos Processos de Produção, Acesso e Disseminação da Informação, Marília, FAPESP, 2006.

Rabikauskas, S. J. P. (2000). Diplomática general. Roma: [s.n.], 2000.
Rayward, W. B. (1994). Visions of Xanadu: Paul Otlet (18681944) and hipertext. // Journal of the American Society for Information Science. 45:4 (May 1994) 235-259.

Rayward, W. B. (1995). Orígenes de la ciencia de la información y del Instituto International de Bibliografía: Federación Internacional de Información y Documentación (FID). // Rayward W. B.; Arnan Rived, P. Hasta la documentación electronica. 2.ed. Mundarnau: Madrid, 1995.

Reis, G. C. (2002). A dimensão diplomática do conteúdo informacional de documentos técnicos: uma análise de manuais de instruções de pulverizadores automotrizes. Dissertação (Mestrado em Ciência da Informação) Faculdade de Filosofia e Ciências. Marília: Universidade Estadual Paulista, 2002.

Riesco Terrero, A. (2000). La Paleografia y Diplomática en el marco de los estudios de Documentación. // I Congreso Universitário de Ciências de la Documentación. Madrid, Universidad Complutense, 2000. http://www.ucm.es/info/multidoc/multidoc/revista/ num10/paginas/pdfs/Ariesco.pdf (2006-2-20).

Rondinelli, R. C. (2002). Gerenciamento arquivístico de documentos eletrônicos: uma abordagem teórica da diplomática arquivística contemporânea. Rio de Janeiro: Ed. FGV, 2002.

Santos, P. De M. dos. (2006). O ponto de inflexão Otlet: uma visão sobre as origens da Documentação e o processo de construção do Princípio Monográfico. Dissertação (Mestrado em Ciência da Informação). São Paulo: Escola de Comunicação e Artes, Universidade de São Paulo, 2006

Sarde, C. L. P. (2003). Análise diplomática de bulas de medicamentos. Monografia (Trabalho de Conclusão de Curso em Biblioteconomia). Marília: Universidade Estadual Paulista Júlio de Mesquita Filho, 2003.

Smit, J. W. (1986). O que é documentação? São Paulo: Brasiliense, 1986. (Coleção primeiros passos, 174).

Smit, J. W.; Guimarães, J.A.C. (1998). Os documentos arquivísticos e seu conteúdo: função $X$ tematicidade. Comunicação apresentada no XII Congresso Brasileiro de Arquivologia, 1998.

Smit, J. W.; Guimarães, J.A.C. (2001). Análise documentária. // Smit, J. W.; Lima, Y. D. Organização de arquivos: XV Curso de especialização. São Paulo, Escola de Comunicação e Artes/USP, 2001. 61-76. (Processamento, 3).

Straioto, A. C. (1997). Análise documentária de embalagens de shampoo: uma alternativa de uso do método diplomático para o profissional da informação. Monografia (Trabalho de Conclusão de Curso em Biblioteconomia). Marília: Faculdade de Filosofia e Ciências, Universidade Estadual Paulista, , 1997.

Tamayo, A. (1996). Archivística, diplomática y sigilografía. Madrid, Cátedra, 1996.

Tessier, G. (1952). La Diplomatique. Paris, PUF, 1952.

Valente, J. A. V. (1978) Acerca do documento. // Revista Brasileira de Biblioteconomia e Documentação. 11:3-4 (1978) 177-198.

Woledge, G. (1983). "Bibliography" and "Documentation": words and ideas. // Journal of Documentation. 39:4 (1983) 266-279. 